\title{
Study on the Preparation of Superabsorbent Composite of Chitosan-g-poly (Acrylic Acid)/Kaolin by In-situ Polymerization
}

\author{
Huafei Xie \\ Institute of Green Chemical Engineering, Zhongkai University of Agriculture and Engineering \\ Guangzhou 510225, China \\ Tel: 86-20-3417-2871 E-mail: zhonghua2344@yahoo.com.cn \\ Zhenyu Jia (Corresponding author), Jinhui Huang \& Cuirong Zhang \\ Institute of Green Chemical Engineering, Zhongkai University of Agriculture and Engineering \\ Guangzhou 510225, China
}

Tel: 86-20-3417-2871Ｅ-mail: gdjiazhenyu@163.com

Received: January 20, 2011 Accepted: March 2, 2011 doi:10.5539/ijc.v3n3p69

This research was financially supported by National Science Foundation of China (20776164) and Key Scientific and Technological Projects of Guangdong Province (2010B011000007).

\begin{abstract}
A novel chitosan-g-poly (acrylic acid)/kaolin (CTS-g-PAA/kaolin) superabsorbent composite was prepared by graft polymerization among chitosan (CTS), acrylic acid (AA) and kaolin in aqueous solution. Effects of polymerization variables, including the content of the weight ratio of crosslinker, initiator, kaolin and CTS to AA, on water absorbency were studied. The results from FT-IR spectra showed that CTS and kaolin participate in graft polymerization reaction with AA. Results indicated that the superabsorbent composite possessed excellent absorbency properties, using $0.3 \% \mathrm{~K}_{2} \mathrm{~S}_{2} \mathrm{O}_{8}$ as initiator and $0.05 \% \mathrm{~N}$, N-methylenebisacrylamide (MBA) as crosslinking agent, when the neutralization degree of AA was $70 \%$, and the ratio of CTS and kaolin to AA was 0.13 and 0.13 , respectively.
\end{abstract}

Keywords: In-situ polymerization, Acrylic acid (AA), Chitosan (CTS), Kaolin, Superabsorbent composite

Superabsorbent composite is a novel polymer materials with high absorbency and good water retention properties, and has been applied in many areas such as health care, water-saving agriculture, materials industries and so on (Chou, 2003, pp.690-691). Due the shortcomings of polyacrylic acid (PAA) superabsorbent such as poor biodegradability, high cost, single features and so on, superabsorbent composite preparation by graft polymerization among natural biodegradable polymers and hydrophilic monomers has been one of the focuses all over the world. CTS is the second largest renewable resources next to cellulose and possesses the properties such as large yield, strong regeneration ability and excellent biological activity. If applied in absorbent resin, it would have been a novel natural functional biological agent in material field (Marguerite, 2006, pp.603-632; Seo, 2004, pp.794-800; Jayakumar, 2005, pp.142-158).

In recent years, preparation of superabsorbent composite by organic-inorganic hybrid methods has attracted extensive attentions (Xie, 2009, pp.129-132; Wang, 2009, pp.731-737), and graft polymerization among inorganic clay minerals and vinyl monomers possessed several advantages such as ameliorating water absorption of resins, enhancing gel strength, lowering production costs and so on (Wang, 2006, pp.12-18). Kaolin belongs to hydrophilic layered silicate mineral, the link between crystal layers is very weak and water or other polar molecules easily enters crystal layer. Consequently, it has strong water absorption, and as its particle surface had many hydroxyl groups and active sites, it could interact with organic polymers to form organic/inorganic hybrid network structure which could efficaciously enhance water absorbency rate and gel strength after absorption. Therefore, the synthesis of chitosan-g-acrylic acid/kaolin (CTS-g-PAA/Kaolin) superabsorbent composite by in situ polymerization was investigated in the present paper. 


\section{Materials and methods}

\subsection{Experimental principle}

By the initiation of ammonium persulfate, hydroxyl in the $\mathrm{C} 3$ position of CTS lost $\mathrm{H}$ atoms to generate free radicals, and began graft reaction with AA and MBA in their carbon-carbon double bonds accompanied by the dehydration esterification reaction between carboxyl of AA and silanol groups of Kaolin. Sodium acrylate-CTS/Kaolin free radicals generated, continued chain growth polymerization with AA, and finally terminated the chain growth (Pourjavadi, 2006, pp.203-211). At the same time, sodium acrylate could also generate free radicals, and began polymerization reaction. By the cross-linking of MBA, cross-linking occurred between PAA and PAA, sodium acrylate-chitosan/Kaolin and sodium acrylate-chitosan/Kaolin, and PAA and sodium acrylate-chitosan/Kaolin, and formed three-dimensional network structure. Main reaction equations were depicted in Figure 1 as follows:

\subsection{Reagents and instruments}

Acrylic acid: Tianjin Fu Chen Chemical Reagent Factory, AR grade; chitosan: Guangzhou Qi Yun Biotechnology Co., Ltd., AR grade; kaolin: Tianjin Guangcheng Chemical Reagent Co., Ltd., AR; ammonium persulfate: Guangzhou Chemical Reagent Factory, AR grade; MBA: Shanghai Johnson Chemicals Co. Ltd., AR grade; $\mathrm{NaOH}$ : Tianjin North Union Fine Chemical Company, AR grade; $\mathrm{NaCl}$ : Tianjin Guangcheng Chemical Reagent Co., Ltd., AR grade; super constant temperature water bath: Changzhou Sino Instrument Co., Ltd.; Fourier transform infrared spectrometer: PerkinElmer Company, USA; vacuum drying oven: Gongyi Yingyu Yuhua Instrument factory; ultrasonic dispersion device: Gongyi Yingyu Yuhua Instrument factory; constant temperature magnetic stirrer: Gongyi Yingyu Yuhua Instrument factory.

\subsection{CTS-g-PAA/Kaolin preparation of superabsorbent composite}

$15 \mathrm{~g}$ AA was added to beaker with cold bath, and $0.2 \mathrm{~g} / \mathrm{mL} \mathrm{NaOH}$ aqueous solution was dropped into the beaker till certain neutralization. After cooling to room temperature, different amount of Kaolin, CTS and cross-ling agent MBA were added and dispersed by ultrasonic dispersion device for $10 \mathrm{~min}$, transferred to super constant temperature water bath at $60{ }^{\circ} \mathrm{C}$, after $\mathrm{N}_{2}$ blanketing for $30 \mathrm{~min}$, and certain amount of APS was added to generate free radicals. After reaction for $90 \mathrm{~min}$, the resultant products were dried in vacuum drying oven for $8 \mathrm{~h}$. The resultant dry bulk composite resin products became samples after grinding and screening.

\subsection{IR analysis}

Kaolin, CTS, PAA, CTS-g-PAA, and CTS-g-PAA/Kaolin were crushed respectively. Samples were assayed using $\mathrm{KBr}$ tablet by Fourier transform infrared spectroscopy Spectrum one from PerkinElmer. Scan scope ranged from 450 to $4000 \mathrm{~cm}^{-1}$, scan times was 8 , and resolution rate was $4 \mathrm{~cm}^{-1}$.

\subsection{Determination of liquid absorbency rate}

Certain amount of dried samples after accurate weighting was placed into home-made tea bags, and added into a beaker containing certain volume of distilled water and $0.9 \% \mathrm{NaCl}$ solution. After swelling and saturation, tea bags were suspended to remove excessive water, and then the whole gel was weighted. Liquid absorbency rate was calculated according to the following formula:

$\mathrm{Q}\left(\mathrm{Q}^{\prime}\right)=\left(\mathrm{m}_{2}-\mathrm{m}_{1}\right) / \mathrm{m}_{1}$

Where Q (Q') represented the liquid (salt or water) absorbency rate $(\mathrm{g} / \mathrm{g})$ of composite $\mathrm{SAR}, \mathrm{m}_{1}$ the dry weight (g) of composite SAR and $\mathrm{m}_{2}$ the wet weight ( $\mathrm{g}$ ) of gel after composite SAR absorbed water.

\section{Results and discussions}

\subsection{Structural analysis of composite resin (Wang, 2006, pp.601-608)}

IR spectra of Chitosan after graft polymerization of Kaolin, CTS, PAA and sodium polyacrylate and PAA-g-chitosan/kaolin composite resin was depicted in Figure 2. Peaks at $1593 \mathrm{~cm}^{-1}$ and $1380 \mathrm{~cm}^{-1}$ were the characteristic absorption peaks of amino in CTS molecule. As seen from Figure 2D and 2E, after grafted with $\mathrm{AA}$, the characteristic absorption peaks disappeared, asymmetric COO- appeared at $1567 \mathrm{~cm}^{-1}, 1453 \mathrm{~cm}^{-1}$ and $1408 \mathrm{~cm}^{-1}$, peaks at $1730 \mathrm{~cm}^{-1}$ of $\mathrm{C}=\mathrm{O}$ stretching vibration absorption and that in amide $\mathrm{I}$ overlapped, and compared to CTS, peaks strength increased significant accompanied by shifting, which explained that AA took the graft polymerization reaction with CTS molecular chain. As seen from Figure 2E, the characteristic absorption peaks of Si-OH in Kaolin at $3676 \mathrm{~cm}^{-1}$ disappeared, its stretching vibration appeared at $1089 \mathrm{~cm}^{-1}$, and antisymmetric stretching vibration absorption peaks of Si-O-Si appeared at $1021 \mathrm{~cm}^{-1}$. IR spectra analysis indicated that AA, CTS and Kaolin participated in graft polymerization together. 


\subsection{Factors affecting reaction system}

\subsubsection{Effects of initiator amount on the swelling and imbibition of resins}

Initiator amount could not only affect the rate, conversion rate and product molecular weight of polymerization, but also whether to implosion during reaction process. Figure 3 illustrated effects of APS on water absorbency rate of CTS-g-PAA/Kaolin composite resin. When initiator amount ranged from $0.1 \%$ to $0.3 \%$, water absorbency rate of composite resin in distilled water and $0.9 \% \mathrm{NaCl}$ solution showed an ascending trend; when increasing initiator amount further, its liquid absorbency rate decreased slightly, which could be ascribed to the facts that due to small amount of initiator, the quantity of free radicals in reaction system was quite small, reaction rate was also small, and it was tough to form network structure, which affected the occurrence of polymerization and cross-linking and thus decreased liquid absorbency rate. With the increasing amount of initiator, free radicals resulted from CTS main chain increased, AA amount that participated in graft reaction with CTS followed, and thus the hydrophilicity of reaction products increased. When initiator amount increased continuously, due to the increment of bimolecular radical coupling and polymerization products, polymers of low molecular weight were obtained, which resulted in the reduction of water absorbency rate of superabsorbent composite. Consequently, the amount of APS should be $0.3 \%$.

\subsubsection{Effects of cross-linking agent amount on the swelling and imbibition of resins}

Based on $15 \mathrm{~g}$ AA, cross-linking agent MBA amount was $0.01 \%, 0.03 \%, 0.05 \%, 0.07 \%$, and $0.09 \%$ (the mass fraction of acrylic acid, the same below), respectively. Result was depicted in Figure 4. With the increasing of MBA, liquid absorbency ability lowered gradually. When MBA amount was less than $0.05 \%$, its distilled water absorbency rate decreased dramatically while $0.9 \% \mathrm{NaCl}$ solution remained unchanged; when larger than $0.05 \%, 0.9 \% \mathrm{NaCl}$ solution rate decreased progressively while distilled water slightly. Due to less MBA (less than $0.05 \%$ ) and cross-linking density, resin became half-aqueous state or part of them became water soluble resin with low water absorbency rate; after absorbing $0.9 \% \mathrm{NaCl}$ solution, the osmotic pressure of salt solution made resin to maintain gel form, and therefore, the liquid absorbency ability changed little. With the increasing of MBA (more than $0.05 \%$ ), cross-linking density increased, effects of osmotic pressure of salt solution on resin lowered, and therefore, liquid absorbency ability in salt solution decreased sharply; the reduction of three-dimensional network pore size resulted in descending of liquid absorbency ability in distilled water.

\subsubsection{Effects of AA neutralization on the swelling and imbibition of resins}

Figure 5 showed effects of AA neutralization on liquid absorbency rate of composite resin. With the increasing of AA neutralization, water absorbency rate of composite resin in distilled water and $0.9 \% \mathrm{NaCl}$ solution increased firstly and decreased finally; when AA neutralization reached $70 \%$, liquid absorbency ability attained the largest level. Monomer neutralization directly affected the hydrophilic group types and charge number in resin molecular chains, and thus affected the water absorbency ability of polymers. When neutralization was less than $70 \%$, reaction was easy to trigger under acidic conditions with high monomer conversion rate. However, excessive low neutralization resulted in the reduction of ionic concentration in resins, and electrostatic repulsion and osmotic pressure of network decreased, which was not conducive to improve water absorbency ability of polymers; on the other hand, excessive high neutralization resulted in metal ionic concentration in resins increased, $\mathrm{Na}^{+}$released from the swelling and imbibition of resins in water could generate counterion shielding effects, and therefore, the swelling and stretching ability of polymer chains decreased, and water absorbency followed. Accordingly, when neutralization was $70 \%$, liquid absorbency rate of resin in distilled water and $0.9 \% \mathrm{NaCl}$ solution reached a higher level.

\subsubsection{Effects of CTS amount on the swelling and imbibition of resins}

Other conditions remain unchanged, based on $15 \mathrm{~g} \mathrm{AA}, 0.5,1.0,1.5,2.0,2.5$, and $3.0 \mathrm{~g}$ CTS was added to participate reaction, respectively. Effects of CTS amount on water absorbency rate on composite resin were investigated, and result was depicted in Figure 6. First, with the increasing of CTS amount, there were many available monomer molecules around CTS large molecular free radical chain that would be grafted onto CTS backbone. Large molecular chain would grow, and network structure of polymers extended and thus formed three-dimensional structure; at the same time, due to the increasing of the hydrophilic hydroxyl contents, hydrophilic properties of composite resins enhanced, and therefore its water absorbency rate increased. When $m$ $(\mathrm{CTS}) / \mathrm{m}(\mathrm{AA})>0.13$, with the increasing of the ratio, water absorbency rate reduced inversely. Water absorbency ability of hydroxyl was far smaller than carboxyl in CTS, and with the increasing of hydroxyl and relative decreasing of carboxyl, liquid absorbency rate decreased. Meanwhile, due to the increment of CTS amount, the molecular weight of side chains after polymerization increased, and it tended to be easy for molecular chains to intertwist. Consequently, cross-linking improved, excessive large system viscosity hindered 
the molecular motion, and liquid absorbency ability of resins decreased. When the ratio of CTS to AA was 0.13 , liquid absorbency rate of resins reached a higher level.

\subsubsection{Effects of Kaolin amount on the swelling and imbibition of resins}

Effects of kaolin amount on the swelling and imbibition of resins was depicted in Figure 7. As seen from Figure 7, when adding small amount of Kaolin (less than $1.5 \mathrm{~g}$ ), liquid absorbency rate of composite resin was lower, and reduced gradually with the increasing of Kaolin amount; When adding larger amount of Kaolin (more than $2.0 \mathrm{~g}$ ), the rate increased significantly compared to less amount, but also reduced with the increasing of Kaolin amount. Kaolin belongs to hydrophilic inorganic minerals, and copolymers cross-linked with hydroxyl and active sites (permanent charge, variable charge and exchangeable cations) in the particle surface of Kaolin. When adding small amount of Kalin, Kaolin played a role as cross-linking agent in composite resins, cross-linking density increased, and the swelling and imbitition ability of composite resins decreased. When adding large amount of Kaolin, although Kaolin belongs to hydrophilic inorganic minerals and possesses certain water absorbency ability, water absorbency rate was still low compared to organic polymer absorbent materials. More amount of Kaolin resulted in the swelling and imbibition of composite resins, and at the same time led to form gaps within resins. Therefore, electrostatic repulsion and osmotic pressure of network increased, and liquid absorbency ascended. When adding excessive amount of Kaolin, the distance between cross-linking points became short, and effective grafting positions in composite resins failed to increase. It was just a mechanical mixture in weight; Furthermore, water absorbency amount was quite low compared to resins, and therefore, water absorbency ability of samples decreased inevitably.

\section{Conclusions}

CTS-g-PAA/kaolin superabsorbent composite was prepared successfully by graft polymerization, and IR spectra verified that AA, CTS and Kaolin participated graft polymerization together. Polymerization variables, including the content of the weight ratio of cross-linking agent, initiator, the weight ratio of CTS to AA, kaolin content and AA neutralization gave certain impacts on water absorbency ability. The optimum experimental parameters were AA neutralization, initiator content, cross-linking agent content, the ratio of CTS to AA, and the ratio of Kaolin to AA as $70 \%, 0.3 \%, 0.05 \%, 0.13$ and 0.13 , respectively. The addition of CTS made the degradation of composite resin feasible, and the induction of Kaolin could lower the costs further and offered chances for its practical application.

\section{References}

Chou, X.X. (2003). Super absorbent. Beijing: Chemistry industry press, 690-691.

Jayakumar, R., Prabaharan, M., \& Mano, J.F. (2005). Graft copolymerized chitosan-present status and applications. Carbohydrate Polymers, 62:142-158.

Marguerite, R. (2006). Chitin and chitosan: properties and applications. Prog. Polym. Sci., 31:603-632.

Pourjavadi, A., \& Mahdavinia, G.R. (2006). Chitosan-g-poly (acrylic acid)/kaolin superabsorbent composite: Synthesis and characterization. Polymers \& Polymer Composites, 14(2):203-211.

Seo, K.W., Kim, D.J., \& Park, K.N. (2004). Swelling properties of poly (AM-co- AA)/chitosan pH sensitive superporous hydrogels. J.Ind.Eng.Chem, 10(5):794-800.

Wang, A.Q., \& Zhang, J.P. (2006). Organic and inorganic composite water superabsorbent polymer. Beijing: Science press, 12-18.

Wang, H.W., Dong, Y.M., \& Zhao, Y.Q. (2006). Advance of graft copolymerization onto chitin and chitosan. Progress in Chemistry, 18(5):601-608.

Wang, Q., Zhang, J.P., \& Wang, A.Q. (2009). Preparation and characterization of a novel pH-sensitive chitosan-g-poly (acrylic acid)/attapulgite/sodium alginate composite hydrogel bead forcontrolled release of diclofenac sodium. Carbohydrate Polymers, 78:731-737.

Xie, Y.T., \& Wang, A.Q. (2009). Synthesis \& water retention of chitosan-g-poly (acrylic acid)/rectorite superabsorbent composites. Polymer Materials Science \& Engineering, 25(7):129-132.

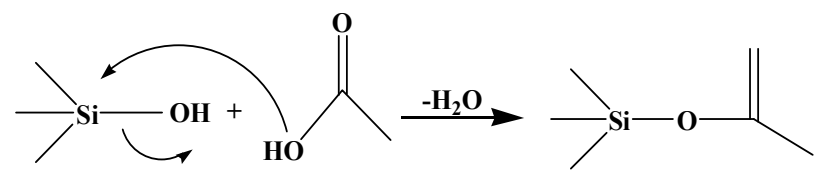




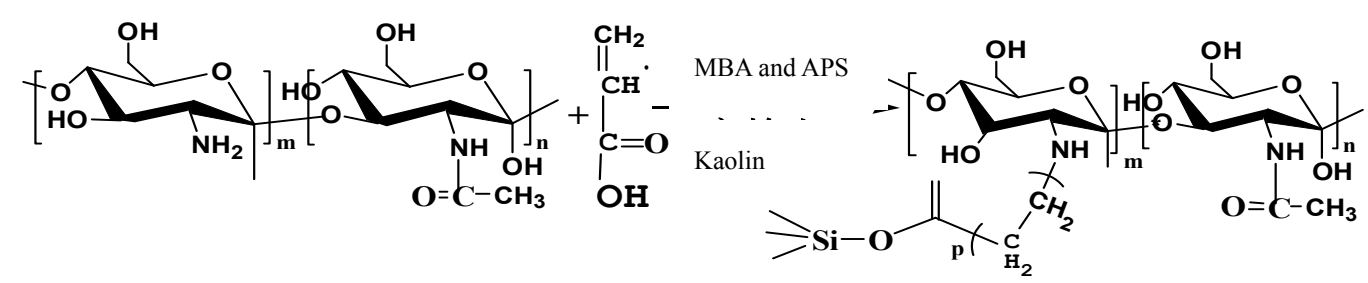

Figure 1. Preparation reaction equations of composite resin

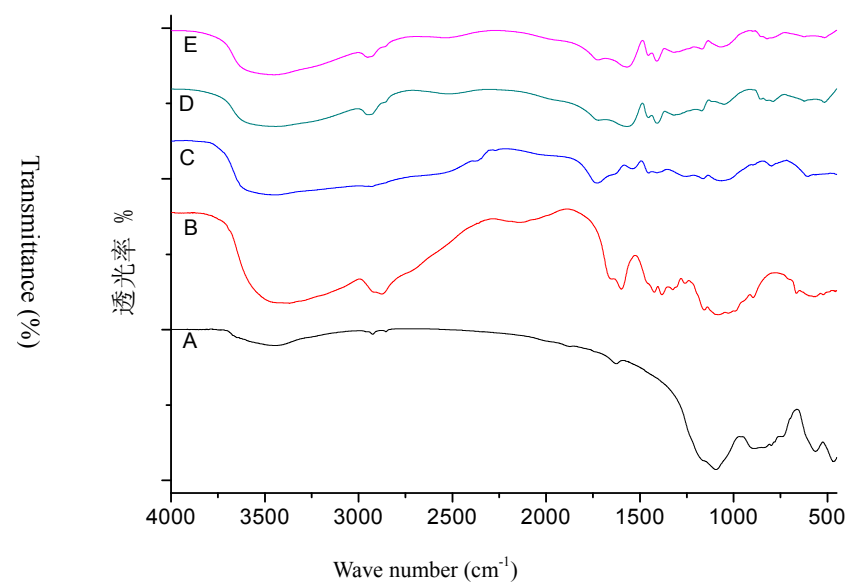

Figure 2. IR Spectra. A, Kaolin; B, CTS; C, PAA; D, CTS-g-PAA; E, CTS-g-PAA/Kaolin

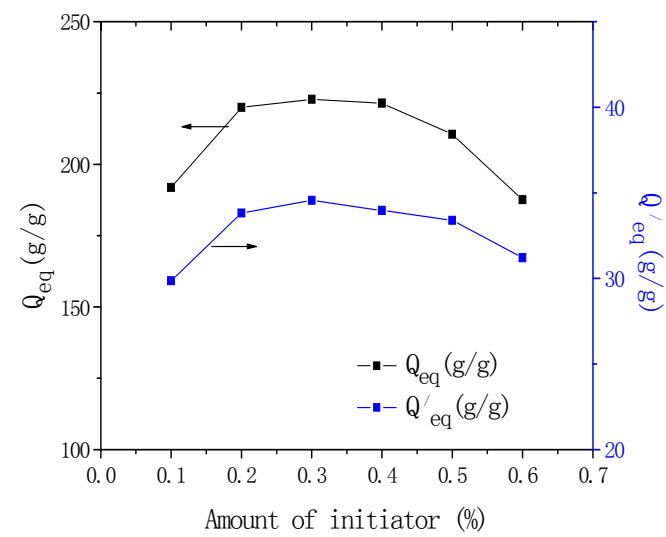

Figure 3. Effects of cross-linking agent amount on water absorbency rate

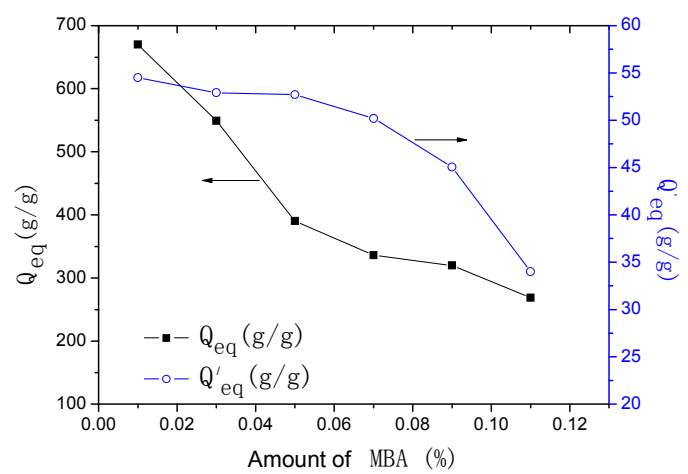


Figure 4. Effects of MBA on water absorbency rate

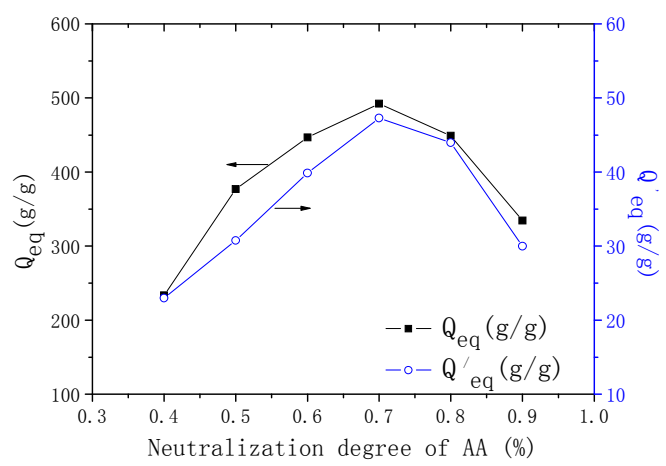

Figure 5. Effects of AA neutralization on water absorbency rate

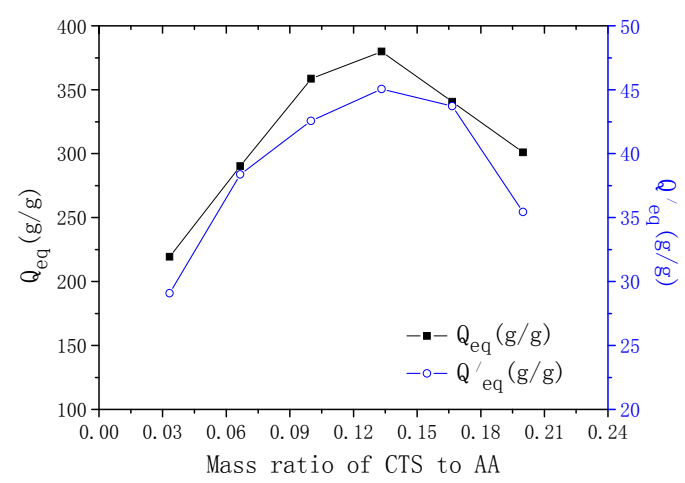

Figure 6. Effects of the ratio of CTS to AA on water absorbency rate

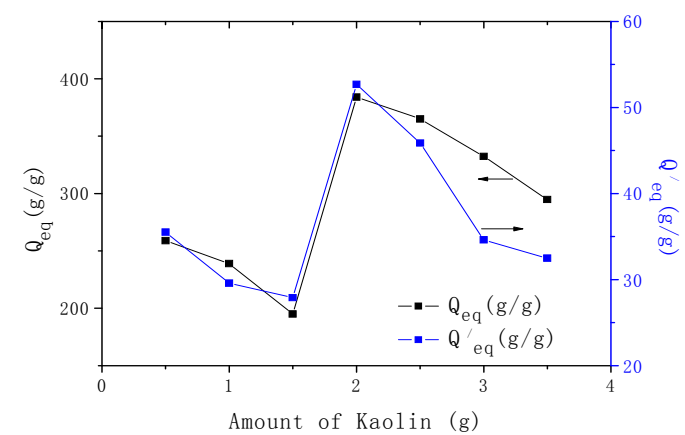

Figure 7. Effects of Kaolin amount on water absorbency rate 